\title{
Correction to: Factors associated with in-hospital mortality and adverse outcomes during the vulnerable post-discharge phase after the first episode of acute heart failure: results of the NOVICA-2 study
}

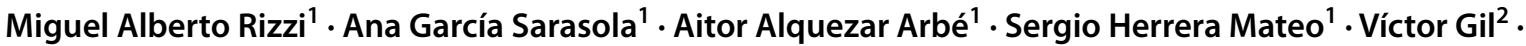 \\ Pere Llorens ${ }^{3}$. Javier Jacob ${ }^{4}$. Francisco Javier Martín-Sánchez ${ }^{5}$. Pablo Herrero Puente ${ }^{6} \cdot$ Rosa Escoda $^{2}$. \\ Begoña Espinosa ${ }^{3}$. Àlex Roset ${ }^{4} \cdot$ Raquel Torres-Gárate $^{7}$. José Torres-Murillo ${ }^{8} \cdot$ Ana B. Mecina $^{9}$. \\ María Pilar López-Díez ${ }^{10}$. José María Álvarez Pérez ${ }^{10}$. Josep Tost ${ }^{11}$. Eva Salvo ${ }^{12}$. María Luisa López-Grima ${ }^{13}$. \\ Cristina Gil ${ }^{14} \cdot$ María Mir $^{15}$. Frank Ruschitzka ${ }^{16}$. Ovidiu Chioncel ${ }^{17}$. Òscar Miró ${ }^{2}$ on behalf of the ICA-SEMES \\ Research Group
}

Published online: 23 December 2020

(c) Springer-Verlag GmbH Germany, part of Springer Nature 2020

\section{Correction to: Clinical Research in Cardiology https://doi.org/10.1007/s00392-020-01710-0}

The original version of this article unfortunately contained a mistake.

A sentence in the acknowledgements was missing. The missing sentence is given below.

The original article can be found online at https://doi.org/10.1007/ s00392-020-01710-0.

Miguel Alberto Rizzi

MRizzi@santpau.cat

1 Emergency Department, Hospital de la Santa Creu I Sant Pau, Barcelona, Spain

2 Emergency Department, Hospital Clínic, University of Barcelona, Barcelona, Spain

3 Emergency Department, Short Stay Unit and Hospitalization at Home, Hospital General de Alicante, Miguel Hernandez University, Alicante, Spain

4 Emergency Department, Hospital Universitari de Bellvitge, Barcelona, Spain

5 Emergency Department, Hospital Clínico San Carlos, Instituto de Investigación Sanitaria Hospital Clínico San Carlos (IdISSC), Universidad Complutense de Madrid, Madrid, Spain

6 Emergency Department, Hospital Universitario Central de Asturias, Oviedo, Spain

7 Emergency Department, Hospital Universitario Severo Ochoa de Leganés, Madrid, Spain

8 Emergency Department, Servicio de Urgencias, Hospital Reina Sofía de Córdoba, Sevilla, Spain
We would like to thank the Department of Medicine of the Autonomous University of Barcelona for its logistical and administrative support.

The original article has been corrected.

9 Emergency Department, Hospital Universitario Fundación Alcorcón, Madrid, Spain

10 Emergency Department, Hospital Universitario de Burgos, Burgos, Spain

11 Emergency Department, Hospital de Terrassa, Barcelona, Spain

12 Emergency Department, Hospital Universitario La Fe, Valencia, Spain

13 Emergency Department, Hospital Doctor Peset, Valencia, Spain

14 Emergency Department, Hospital Universitario de Salamanca, Salamanca, Spain

15 Emergency Department, Hospital Infanta Leonor, Madrid, Spain

16 Department of Cardiology, Heart Failure Clinic and Transplantation, University Heart Centre Zurich, Zurich, Switzerland

17 Emergency Institute for Cardiovascular Diseases 'Prof. C.C. Iliescu', University of Medicine Carol Davila, Bucharest, Romania 